long will it be before the virus changes sufficiently to evade the immune barrier which the human herd has raised against it ?

Attempts to produce the "next" variant in laboratory animals have not been successful. ${ }^{11}$ In recent years it has become clear that influenza $A$ and $B$ viruses can in fact be isolated from cases of respiratory disease between epidemics, in periods when clinical or epidemiological observations such as the frequency of deaths from pneumonia, sickness claims, or outbreaks in institutions or local communities do not show that an epidemic is in progress. Thus, though the virus may seem to have disappeared, it can be found if looked for sufficiently thoroughly. However, so far no new serotype has been caught in the act of antigenic evolution.

An even less predictable event is the appearance of radically new influenza viruses, such as the Asian influenza virus. For many years it was thought that they might come from an animal reservoir. Unfortunately, in the best-studied example, that of influenza in American pigs, it seemed more likely that the virus had gone from man to pig in the 1918 epidemic rather than the reverse. But it does now provide a good example of an influenza virus which maintains itself in animals. Its life cycle, involving earthworms and nematodes, is rather extraordinary, but it is apparently independent of man-except that it is man who keeps the pigs. However, in recent years we have learnt much more about influenza viruses in animals. It was discovered in 1955 that the longknown fowl plague virus is actually an influenza $A$ virus, ${ }^{12}$ and since then that there are also other antigenically distinct influenza A viruses affecting ducks, ${ }^{13}$ turkeys, terns, and game birds, and two different serotypes have been recovered from horses both in Europe ${ }^{14}$ and in North America. ${ }^{15}$ Human beings have been infected experimentally with one of the horse viruses, but large doses were used and only one volunteer became sick. ${ }^{16}$

Antibody against one of the horse viruses has been found in the sera of old persons. ${ }^{17} 18$ It is difficult to interpret this. But if man was infected in the last century with a virus related to one now infecting horses, that does not prove that it came from horses or from any other animal. Furthermore, there is no well-documented instance of an epidemic beginning in animals and spreading to man, though it might be a rare event and also take place in economically impoverished places where man and animals live close together and opportunities for precise observation are few. There are examples of almost completely new antigenic serotypes of influenza $\mathbf{B}$ which produced local outbreaks of disease in man but did not spread across continents as they were expected to do. ${ }^{1920}$ They apparently lacked something, perhaps the ability to survive in air and so spread rapidly, which would have made them into completely successful epidemic strains. So animal influenza viruses which would ordinarily grow rather poorly in the cells of man would, if the theory were correct, be likely to produce a few cases more often than a distinct epidemic. Any possible examples should be quickly reported and investigated by expert virologists.

Recent experiments on mice ${ }^{2122}$ have shown that the virus is transmitted as airborne droplets, and that the rate of transmission can be reduced by increasing ventilation and modified by the temperature and humidity of the air. Some viruses are much better "spreaders" than others, though it is still not clear why they are. Further experiments, just described, ${ }^{23}$ show that though serious disease can be prevented by vaccinating mice with live or killed vaccine the live vaccine also reduces the ability to transmit infection to other animals when mice are reinfected, while mice protected with killed vaccine can still act as transmitters.

The epidemiology of influenza is rather like the study of economics: it does not tell us what is going to happen so much as why things happen after they have done so. Consequently a prediction for the coming winter in Britain must be based on a balance of probabilities. Two years ago we had influenza $A 2$ and $B$, so we might expect influenza A2 to return this year, as it seems to have done in the southern hemisphere during our summer. There is no sign of a radically new antigenic type of virus, and many people carry antibodies against the current types. So if an epidemic comes it is likely to be mild.

\section{Postoperative Gas Gangrene}

It has recently been reported in the daily press that two cases of gas gangrene, one of them fatal, have followed amputation through the thigh in a Birmingham hospital, and that an operating-theatre suite has consequently been closed. Some comments on this occurrence ${ }^{1}$ refer to the ubiquity of pathogenic bacteria in the hospital environment and the necessity for greater and very expensive precautions in order to reduce the risk so entailed. A further report ${ }^{2}$ concerns three cases of gas gangrene at a London hospital, but gives no information about the circumstances in which they arose.

No lessons can be drawn from these two episodes until a full account of them is available, but there are certain facts about the causation of this infection, and particularly with regard to its source, which apparently deserve to be more widely known. The organism almost invariably causing postoperative gas gangrene is Clostridium welchii. It is a normal inhabitant of the bowel of man and animals, and almost always to be found in soil, dirt, and dust. Its spores are capable of prolonged survival. Their exclusion from the hospital environment is impossible. For instance, E. J. L. Lowbury and H. A. Lilly ${ }^{3}$ found them in every sample of air examined in a hospital, including that of operatingtheatres. Moreover, the organism is not uncommonly present in the exudate from traumatic wounds or burns which are the seat of nothing more than transient suppuration. It is thus implanted in many traumatic wounds and probably in a substantial proportion of surgical wounds without giving rise to the acute necrosing myositis with formation of gas which is gas gangrene.

What, then, is the missing factor which ensures these patients' safety ? It is simply that the tissues involved have an adequate blood supply and thus an oxygen tension in which clostridia cannot grow. The spores of an anaerobe cannot even germinate in a tissue normally supplied with blood. In traumatic gas gangrene the infection originates in muscle which has been crushed or cut off from its blood supply. Only profuse growth of clostridia in such an area can create conditions in which normal muscle can subsequently be invaded.

For three reasons gas gangrene is more likely to follow thigh amputation for obliterative arterial disease than other

\footnotetext{
Observer, 1 October 1967.

2 The Times, 5 October 1967.

s Lowbury, E. J. and Lilly, H. A. 7. Hyg. (Lond.), 1958, 56, 169.

- Williams, R. E. O., Blowers, R., Garrod, L. P., and Shooter, R. A. Hospital Infection, 2nd d., 1966. London.

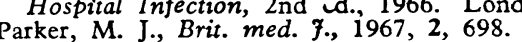

6 Price, D. J. E., and Shooter, R. A., ibid., 1964, 2, 1176.
} 
operations. Firstly, the incision must be through skin not far distant from the anus, and intestinal bacteria are more widely distributed over this area than is commonly realized; among them the spores of clostridia are resistant to ordinary methods of skin disinfection. Secondly, the operation entails severing large masses of muscle in which bacteria from the region of the incision can be directly implanted. Thirdly, and most important, the disease which has necessitated the operation reduces the blood supply to the area, and may thus reduce the oxygen tension to a level permitting clostridial growth. The degree to which this area is so affected is doubtless one variable factor determining the outcome. Others are the efficacy of skin cleansing and the toxigenicity of the patient's own strain of $\mathrm{Cl}$. welchii. This is a property in which different strains vary widely.

This operation carries the imperative indication for penicillin cover in doses allowing for the fact that clostridia are less sensitive to penicillin than, for instance, streptococci. At least 500,000 units should be given at intervals of not more than six hours for seven days." Other operations with a similar risk meriting penicillin cover were recently referred to in our correspondence columns by $\mathrm{M}$. J. Parker. ${ }^{5}$

Gas gangrene is a rare and terrifying condition, and it is perhaps natural that operating-theatre staffs should regard it as calling for exceptional measures. In fact it is much easier to disinfect a theatre after a case of gas gangrene than after a dirty operation on the lower bowel, because in the former the organisms are all in the vulnerable vegetative form, whereas in faeces they exist largely as resistant spores. That most strains from the normal bowel are as toxigenic as those which have had the opportunity of causing gas gangrene has been shown by D. J. E. Price and R. A. Shooter. ${ }^{6}$ It remains to be seen whether conditions in the operating-theatres concerned in these recent episodes are considered to require improvement. What can be said with some confidence is that when gas gangrene follows thigh amputation there is no need to look any further for the source of infection than the patient's anus.

\section{Standardization in Human Cytogenetics}

The explosion in our knowledge of human chromosomes and their abnormalities which has occurred during the past ten years has resulted in many difficulties, particularly of communication and nomenclature. There have now been three conferences to consider these problems. The first of these was in Denver in 1960, the second in London in 1963, while the third and last was convened at the University of Chicago in September 1966. The aim of each of these meetings has been to improve communication and understanding in the field of human cytogenetics. At Denver order was brought into the system of numbering human chromosomes, and the international system agreed at that meeting has remained essentially unaltered since. In London the Denver system was consolidated and the alternative use of letters to classify the different chromosome groups, which had been advocated by some workers since 1961, was agreed internationally to be a useful alternative to the Denver system of nomenclature. At the conference it became clear that little progress had been

1 Chicago Conference: Standardization in Human Cytogenetics. Birth Defects, Original Article Series, 1966, Vol. 2, No. 2, New York. Copies can be obtained free from the Medical Department, The National Foundation-March of Dimes, 800 Second Avenue, New York, N.Y. 10017 . made in chromosome identification over the three preceding years.

The report of the Chicago Conference, published recently, is perhaps one of the most important documents published in the field of human cytogenetics. It considers new means for describing the normal chromosomes and deviations from the normal complement, and proposes an entirely new system of notation for the uniform description of the human chromosome set and its abnormalities. This shorthand system is also applicable to the description of the somatic chromosomes of non-human species, so that perhaps from this conference a universal system of notation may eventually develop. The system is most carefully designed so that it should be adaptable to automated data processing and information retrieval systems, which are becoming more and more widely used.

Other topics discussed at the meeting included the recording of data, and recommendations are made in the report on the essential information required if accounts of chromosome abnormalities and variation are to have maximum value. The place of autoradiography in chromosome identification was considered as well as problems related to the automation of chromosome analysis, population cytogenetics-and in particular the types of population suitable for study and the information which might be derived from such studiesand the effects of radiation and mutagenic substances on chromosomes.

The conference needs following up in two important respects. Its recommendation "that in view of the expansion in human cytogenetics during the past six years it is now desirable to explore the feasibility of establishing a central organization for collecting and analysing data on the variability of the human karyotype" should be acted on by the appropriate international and national bodies. Secondly, its report is available. If investigations and journals publishing papers in this and related fields would use the system of notation recommended in the report then the clarity of published work would be considerably improved. In the past there has been confusion, and valuable data have been lost by the publication of inadequate and incomplete case reports. Widespread acceptance of the recommendations of the Chicago conference should make this a thing of the past.

\section{Health Priorities}

The Ministry of Health's team visiting the U.S.A. and Canada to persuade British medical emigrants to return is now at work. Can it offer the attraction that conditions are so different from what they were, or what the emigrants thought they were, that some will be tempted to return? The Ministry's views, as expressed by its Chief Medical Officer, Sir George Godber, in his annual report, ${ }^{1}$ are robustly hopeful of better times ahead for doctors working in both hospital and general practice. That the department has its views also on what the profession should be doing is apparent from Sir George's observation that, "Given an acceptable and workable opportunity ... the medical profession has shown its ability to work constructively and successfully with the department."

In his introduction Sir George dwells on the year he is reviewing, 1966, as one that " may well be seen in retrospect

1 On the State of the Public Health, The Annual Report of the Chief Medical Officer of the Ministry of Health for the Year 1966. 1967. H.M.S.O.

${ }^{2}$ Management Functions of Hospital Doctors, Ministry of Health, 1966. H.M.S.O. 